\title{
Effectiveness of sodium fluoride varnish to prevent dental caries in school children between age of 6 and 7 years: A randomised controlled trial in a low resource setting
}

\section{Rasika Manori Jayasinghe ( $\square$ manorija@pdn.ac.lk)}

Faculty of Dental Sciences, University of Peradeniya

Sameera Senanayake

Queensland University of Technology

Sanjeewa Kularatna

Queensland University of Technology

\section{Research article}

Keywords: clinical trial, fluoride varnish, dental caries, caries prevention, effectiveness

Posted Date: November 4th, 2019

DOl: https://doi.org/10.21203/rs.2.16745/v1

License: (c) (i) This work is licensed under a Creative Commons Attribution 4.0 International License.

Read Full License 


\section{Abstract}

Background Dental caries among children is a significant public health problem world over with multiple factors playing a major role as risk factors in initiation and contnuation of the disease. Fluoride varnish application is an effective intervent in preventing dental caries. Objective of this study was to evaluate the effectiveness of six monthly application of sodium fluoride varnish in prevention of dental caries in deciduous teeth, permanent incisors and molars in children between six and seven years in a low resource setting, Sri Lanka. Method This was a double-blind, randomized controlled trial (NCT02877888). The study population was school children aged six to seven years, living in a district of Sri Lanka. Recruited children were randomly assigned to either a treatment $(n=161)$ or to control group $(n=162)$ using block randomization. Children in the treatment group received fluoride varnish professionally applied every 6 months combined with counselling on maintenance of oral hygiene and diet for a period of 2 years. The control group was only subjected to counselling on maintenance of oral hygiene and diet. Caries examinations were conducted using the International Caries Detection and Assessment System (ICDAS). The primary outcome studied was the number of new caries in the two study groups. Results At the baseline assessment, the propotion of students with dental caries was higher in the intervention groupl (80.9\%) compared to the control group (48.4\%). Similarly the mean number of teeth with dental caries in a student was higher in the intervention group (1.35). At each time interval, the mean number of new dental caries was significantly lower in the intervention group. When independent effect of the intervention on development of new dental caries was assessed, application of fluoride varnish every 6 months for a period of 2 years was significantly associated with less number of new dental caries compared to controls. Conclusion Implementation of fluoride varnish application in a low resource setting is an effective method of preventing dental caries among children. A national level programme would contribute to the reduction of prevalence of dental caries in this age group. Trial registration: NCT02877888

\section{Background}

Dental caries remains a significant public health problem with multiple factors such as socioeconomic, dietary and microbiological playing a major role as risk factors in initiation and continuation of the disease. It is considered the most common cause of toothache and hospital admissions in young children ${ }^{(1)}$. Dental caries in school children not only causes pain, but also affects school attendance, parents' work hours, children's ability to eat, play, rest and sleep. Therefore, dental caries when present significantly reduces the health related quality of life of a child(2).

Improving oral health has been considered as an objective in preventive dental care for children ${ }^{(3)}$. Therefore the measures taken to prevent dental caries should include bacterial biofilm control, dietary counselling, use of fluoride products such as toothpastes, mouth rinses, varnishes, gels and foams, and Caesin Phospho Peptide- Amorphous Calcium Phosphate (CPP-ACP) cream and gums and placing resin- 
based/glass-ionomer pit and fissure sealants.) Professional fluoride varnish application has become popular among all methods as variety of studies has discussed its effect in reducing dental caries ${ }^{(4-6)}$.

Fluoride varnish use in United States has increased markedly since it was approved by the Food and Drug Administration(FDA) in 1994. Fluoride varnish's effectiveness in caries prevention, ease of application, and safety gives it an advantage over other types of topical fluoride treatments (such as gels and rinses) or other methods of dental caries management. As a result, it is regarded as one of the superior topical fluoride agents for young children ${ }^{(7)}$. Fluoride varnish also prolongs the contact time between fluoride and tooth surface to reduce caries. Its active ingredient is usually $5 \%$ sodium fluoride, or $22,600 \mathrm{ppm}$ fluoride. It also has recently gained much attention in dentistry because the risk of dental fluorosis is found to be minimal. The simplicity of its application makes it very suitable and practical for use in different places and groups ${ }^{(8)}$.

Over the last decade, the dental caries experience among six and seven years old school children remain standing at a very high level in Sri Lanka. Results of the most recent national oral health survey highlights that prevalence of dental caries among this age group in the country is $65.5 \%\left({ }^{(9)}\right.$. However, the figures are quite higher when compared to countries in the region such as India ${ }^{(10)}(52 \%)$ and in developed countries such as United Kingdom ${ }^{(11)}$ and United States of America ${ }^{(12)}$, which highlights the need of a strong low cost intervention program which can be implemented at low resource settings island wide to minimize the burden of the dental caries among this age group.

The simplicity of fluoride varnish application makes it very suitable and practical for use in dental clinics and outreach dental services in Sri Lanka, especially in young children and in other special needs groups. Therefore, a study on effectiveness of fluoride varnish application on teeth, among children in a developing country like Sri Lanka will be beneficial in identification of advantages of fluoride varnish application. Furthermore, it would contribute to the improvement of national dental health services in a country like Sri Lanka which operates with a limited financial and human resources.

In this milieu, the main objective of this study was to evaluate the effectiveness of six monthly application of sodium fluoride varnish in prevention of dental caries in deciduous teeth, permanent incisors and molars in children between six and seven years in Sri Lanka.

\section{Methods}

This was a double-blind, randomized controlled trial. The study has been registered with www.clinicaltrials.gov (NCT02877888). The study was undertaken with the understanding and written consent of each participant and according to the principles of clinical trials registry.

The study population was school children aged six to seven years, living in Kurunegala district. The inclusion and exclusion criteria used in the study are described below. The study was conducted at 
Maliyadeva Adarsha Vidyalaya and Wayamba Royal college, Kurunegala from month year to month year. The sample was selected according to below criteria;

Inclusion Criteria:

- Children with at least one permanent tooth present.

- Age 6 to 7 years.

- Residing in Kurunegala municipal council region and tap water is consumed (fluoride levels in drinking water was considered similar).

Exclusion Criteria:

- Children with ulcerative gingivitis and stomatitis. (These children were referred for treatment.)

- No permanent tooth present or stainless steel crowns only.

The students meeting the inclusion and exclusion criteria was randomly assigned, using block randomization in blocks of four, using a computer generated set of random numbers, to either varnish ( $5 \%$ Sodium Fluoride) or control group. A set of random numbers was used to create an allocation sequence, which was contained in individual opaque envelopes for used by the chief investigator. As the patients were recruited, the next envelope in the sequence was opened and the student was assigned to the stated group. All randomisation, sequence generation, and preparation of group allocation materials was performed by a third party who had no direct contact with the clinical aspects of the trial.

The sample size was calculated as 160 each arm to detect $5 \%$ caries change with $80 \%$ power. All children in both groups were provided with a self-administered pre tested questionnaire to assess the quality of life associated with presence of dental caries. Further, all the children received routine caries preventive measures of oral hygiene instructions, dietary advice and professional cleaning. Clinical examinations of all children were performed at the beginning of the study and 1 year and 2 years later. All the caries at the dentinal level of both groups were stabilized with temporary restorations at the beginning. Both varnish and control groups were subjected to use of fluoride tooth paste of $1000 \mathrm{ppm}$. Children in the intervention group received fluoride varnish professionally applied after prophylaxis every 6 months combined with counselling on maintenance of oral hygiene and diet for a period of 2 years. The control group was only subjected to counselling on maintenance of oral hygiene and diet.

During the period of two years, a team visited each school four times at six months' interval for recruitment, dental examinations, and fluoride varnish applications. Trained interviewers collected data on oral health habits and sociodemographic characteristics of the children. Caries examinations were conducted using the International Caries Detection and Assessment System (ICDAS).

The primary outcome studied was the number of new caries in the two study groups. Students and the interviewers were blinded to the intervention (varnish application or control) and they were blinded to the hypothesis, which was investigated in this study. 
The mean number of caries between different groups, segregated according to the variables assessed in the study, were compared. The mean number of caries in a particular group was calculated by dividing the total number of caries in a particular group by the total number of students in that group. As the number of caries showed a skewed distribution, Mann-Whitney U test was used compare the groups. Level of significance was considered as $p<0.05$.

The effect of intervention was measured by the mean number of new caries in different groups. The number of new caries in the usual care and intervention group was calculated at each time point of the survey. The mean number of new caries between the two groups were compared using Mann-Whitney $U$ test. To assess the independent effect of the intervention on the incidence of caries, when other factors are controlled, multiple linear regression was applied. Number of new caries was considered as the dependant variable while student's age, mothers education status, household income status, intervenion/ control status and presence of toothach at the baseline were considered as independant variables. Separate models were prepared each of the three time points.

\section{Results}

The total sample consisted of 321 students, with 161 and 162 in control and intervention groups respectively. Comparison of different characteristics between the two groups is indicated in table 1 . Age distribution $(p=0.05)$, the number of students with current toothache $(p=0.099)$ and the number of students who had dificulty in takng sweets atthe begining of the study $(p=0.102)$ were similar beteen the control and the intervention group. Among those who had toothache at the begining of the study, the duration of pain was similar between the control and the intervention group $(p=0.768)$.

Percentage of students with at least one dental caries at the beginning of the study and the mean number of teeth with dental caries were compared between the control and the intervention group (Table 2). Propotion of students with dental caries was higher in the intervention groupl (80.9\%) compared to the control group (48.4\%). Similarly the mean number of teeth with dental caries in a student was higher in the intervention group (1.35).

Association between sodium fluoride varnish application and the mean number of new dental caries at 6 months, 1 year and 2 years' review are shown in Table 3. At each time interval, the mean number of new dental caries was significantly lower in the intervention group. To assess the independent effect of the intervention on development of new dental caries, when other factors are controlled, multiple linear regression was applied. Application of vanish was significantly associated with less number of new dental caries at two time points (May 2015 and Nov 2016) of the survey (Table 4).

\section{Discussion}

To the authors knowledge, our study was the first randomized control study to assess the effectiveness of vanish application in a developing country. Results indicated that application of vanish was 
significantly associated with less number of new dental caries among children.

Despite the availability of free public dental health service in Sri Lanka, the prevalence of dental caries among school children remains at a significantly high level. According to the results of National Oral Health Survey in 2002/3, the prevalence of dental caries among 6-7 years old (similar age group as in our study), was $65.5 \%{ }^{(9)}$. Variety of low cost sugar containing confectionary products and beverages are flowing into the market which have contributed a lot for these high levels ${ }^{(13)}$. Therefore, primary preventive approach would be the best in order to achieve the success in caries preventive programs.

Almost every country attempts in prevention of dental caries in their young population as it is cost effective, easy achievable and a long lasting measure. Developing countries such as Sri Lanka have planned community based caries preventive programs aiming to cater a large number of population in one setting which is more appropriate with the available limited infrastructure and less amount of money allocated for the maintenance of health care. Therefore, it is important to investigate into effectiveness of such different caries preventive measures to find out the most suitable approach for our population by considering the factors such as cost effectiveness, ease of use, appropriateness for a large population in one setting and high safety.

Although a strict randomization protocol was followed during the study, the intervention group had a significantly higher prevalence of students with dental caries and higher mean number of teeth with dental caries compared to the control group. Thus, if the intervention is not effective among the study population, one would expect the number of new caries also to be higher among the intervention group after the stipulated follow up period. However, our results indicated that application of vanish was significantly associated with less number of new dental caries among the intervention group, highlighting high effectiveness of the intervention.

Efficiency of different caries preventive programs have been investigated worldwide, some with comparison of different approach. A systematic review by James et al in 2010 had reported that caries increment measured radiographically was slightly higher than use of fluoride varnish. However, it was reported that there was no statistical significance in the results ${ }^{(14)}$. A group of researchers in Sweden had carried out a three year-randomized controlled trial as a school based fluoride varnish program using a large sample of seven hundred and eight children. The study sample was categorized as high, medium and low caries risk area by taking oral health survey results into consideration. They had shown that the prevention fraction of fluoride varnish application in high caries risk area was $69 \%$ compared to the control group (15). The conclusion was drawn as six monthly application of fluoride varnish is effective in reduction of caries in high and medium risk areas. However, it is always valuable to investigate the same in our population as the socioeconomic levels are less when compared with Sweden and considering the restricted budget for health care work in our setting. Although it is the most appropriate measure to assess the effectiveness of a health care program, a randomized controlled trial carried out for use of fluoride varnish in Sri Lankan setting could not be found in the literature. 
Another study which had assessed cost effectiveness of fluoride varnish against pit and fissure sealants highlighted that since the use of fluoride varnish hardly requires high levels of infrastructure, it can be more readily applied in nonclinical setting ${ }^{(16)}$. We also consider that studying on use of fluoride varnish is more appropriate to our country in implementing caries preventive programs in non-clinical settings due to the availability of low physical resources for us. The results of our study had shown that mean caries reduction with use of fluoride varnish for six months, one year and two years is $0.94,1.69,1.50$ compared to $1.79,1.77,1.97$ in the control group and the difference was statistically significant. By considering such background, the results of this study would provide a platform to implement programs of fluoride varnish application in community and school based settings thereby contribute to the reduction of prevalence of dental caries in our school children. Therefore, we believe that our findings will also support the relevant health authorities in the country to consider fluoride varnish application when planning national school dental health promotion programs. When carrying out the analysis of the results of our study, relevant ccontributory factors for dental caries such as parents' education levels, parents' occupation, monthly income of the family, number of children at home, frequency of brushing, use of fluoridated tooth paste were also controlled as they might affect the actual effect of intervention.

\section{Limitations}

Although, the caries contributory factors such as amount of sugar intake were considered, the study had to be dependent on the patient response and there may be variations of the sugar intake in intervention and control groups. Although we considered the fluoride levels in drinking water as equal in our sample as they all are from same residential area, there may be differences, which could have altered the results we obtained.

\section{Conclusion}

It could be concluded that presence of dental caries could affect the quality of life in the means of chewing ability and sensitivity to hot and cold food in children aged 6-7 years. Professional application of fluoride varnish every 6 months for a period of 2 years results in significant reduction in dental caries in children of 6-7 years compared to non-applied ones of same age group. Therefore, implementation of fluoride varnish application programs at National level would contribute to the reduction of prevalence of dental caries in the age group of 6-7 years.

\section{Trial status}

This randomized clinical trial has been registered in ClinicalTrials.gov (U.S.) under the registration number NCT02877888. The study adheres to CONSORT guidelines.

\section{Abbreviations}

International Caries Detection and Assessment System (ICDAS) 
Caesin Phospho Peptide- Amorphous Calcium Phosphate (CPP-ACP)

Food and Drug Administration(FDA)

\section{Declarations}

\section{Ethics approval and consent to participate}

The study has been registered with www.clinicaltrials.gov (NCT02877888). The study was undertaken with the understanding and written informed consent of parent/ guardian of all the participants and according to the principles of clinical trials registry. Ethical clearance was obtained from the Ethical Review Committee, Faculty of Dental Sciences, University of Peradeniya, Sri Lanka.

Consent to publish

Not applicable

\section{Availability of data and materials}

The datasets used and/or analysed during the current study are available from the corresponding author on reasonable request.

\section{Competing interests}

The authors declare that they have no competing interest.

\section{Funding}

The authors declare that there was no funding source.

\section{Authors' Contributions}

RMJ (first author) designed the study, carried out with the support of the hospital staff and outlined the first draft of the study. SS (second author) contributed in designing and carrying out the study. He also contributed in statistical analysis and improving the final draft of the article. SK (third author) contributed in outlining the research proposal, analyzing results and improving final draft of the article. He also contributed in supervising the whole process of the study up to submission of the article. All authors read and approved the manuscript.

\section{Acknowledgements}

The authors would like to acknowledge the dental surgeons and the other staff at the Restorative Dentistry unit, Teaching Hospital Kurunegala for the kind support in data collection. Parents and 
caregivers of the children included in the study, Directors of Education, Kurunegala, Principals and teaching staff of Maliyadeva Adarsha Vidyalaya and Wayamba Royal college and all others who have supported for the study are also acknowledged.

\section{References}

1. Pediatrics AAP. Policy on early childhood caries (ECC): classifications, consequences, and preventive strategies. Pediatric dentistry. 2008;30(7 Suppl):40.

2. Tickle M, Milsom KM, Donaldson M, Killough S, O'Neill C, Crealey G, et al. Protocol for Northern Ireland Caries Prevention in Practice Trial (NIC-PIP) trial: a randomised controlled trial to measure the effects and costs of a dental caries prevention regime for young children attending primary care dental services. BMC Oral Health. 2011;11(1):27.

3. Lewis CW, Johnston BD, Linsenmeyar KA, Williams A, Mouradian W. Preventive dental care for children in the United States: a national perspective. Pediatrics. 2007;119(3):e544-e53.

4. Gugwad SC, Shah P, Lodaya R, Bhat C, Tandon P, Choudhari S, et al. Caries prevention effect of intensive application of sodium fluoride varnish in molars in children between age 6 and 7 years. $J$ Contemp Dent Pract. 2011;12(6):408-13.

5. Greig V, Conway DI. Fluoride varnish was effective at reducing caries on high caries risk school children in rural Brazil. Evidence-based dentistry. 2012;13(3):78.

6. Liu BY, Lo E, Chu C, Lin H. Randomized trial on fluorides and sealants for fissure caries prevention. Journal of dental research. 2012;91(8):753-8.

7. Chu C, Lo E. A review of sodium fluoride varnish. General dentistry. 2006;54(4):247-53.

8. Chu C, Lo E. Uses of sodium fluoride varnish in dental practice. Annals of the Royal Australasian College of Dental Surgeons. 2008;19:58.

9. Ministry of Health SL. National Oral Health Survey. Ministry of Health, Sri Lanka 2003.

10. Bali R, Mathur V, Talwar P, Chanana H. National oral health survey and fluoride mapping 2002-2003 India. New Delhi: Dental Council of India. 2004;132.

11. Digital N. Child Dental Health Survey 2013, England, Wales and Northern Ireland: NHS Digital; 2015 [Available from: https://digital.nhs.uk/data-and-information/publications/statistical/children-sdental-health-survey/child-dental-health-survey-2013-england-wales-and-northern-ireland.

12. Dye BA, Thornton-Evans G, Li X, lafolla TJ. Dental caries and sealant prevalence in children and adolescents in the United States, 2011-2012. 2015.

13. Dimitropoulos Y, Holden A, Gwynne K, Irving M, Binge N, Blinkhorn A. An assessment of strategies to control dental caries in Aboriginal children living in rural and remote communities in New South Wales, Australia. BMC oral health. 2018;18(1):177. 
14. James $P$, Parnell $C$, Whelton $H$. The caries-preventive effect of chlorhexidine varnish in children and adolescents: a systematic review. Caries research. 2010;44(4):333-40.

15. Sköld UM, Petersson LG, Lith A, Birkhed D. Effect of school-based fluoride varnish programmes on approximal caries in adolescents from different caries risk areas. Caries research. 2005;39(4):273-9.

16. Neidell M, Shearer B, Lamster IB. Cost-effectiveness analysis of dental sealants versus fluoride varnish in a school-based setting. Caries research. 2016;50(Suppl. 1):78-82.

\section{Tables}

Table 1 : Comparison of percentage number of students, between different variables at the beginning of the study

\begin{tabular}{|l|r|r|r|r|r|}
\hline \multirow{2}{*}{ Characteristic } & \multicolumn{2}{l|}{$\begin{array}{l}\text { Control group } \\
(\mathrm{N}=161)\end{array}$} & \multicolumn{2}{l|}{ Intervention group (N=162) } & \multirow{2}{*}{ p value } \\
\cline { 2 - 6 } & $\mathrm{N}$ & \multicolumn{1}{l|}{$\%$} & $\mathrm{~N}$ & $\%$ & \\
\hline Age & & & & & \\
\hline 6 years & 96 & 59.6 & 79 & 48.8 & 0.050 \\
\hline More than 6 years & 65 & 40.4 & 83 & 51.2 & \\
\hline Difficulty in chewing & 57 & 35.4 & 36 & 22.2 & 0.009 \\
\hline Difficulty chewing vigorously & 48 & 29.8 & 31 & 19.1 & 0.026 \\
\hline Difficulty taking cold or hot food & 48 & 29.8 & 32 & 19.8 & 0.036 \\
\hline Difficulty taking sweets & 42 & 26.1 & 30 & 18.5 & 0.102 \\
\hline Current toothache & 41 & 25.5 & 29 & 17.9 & 0.099 \\
\hline Of those who have current toothache & & & & & \\
\hline Duration (<1 month) & 06 & 14.6 & 05 & 17.2 & 0.768 \\
\hline Duration (>1 month) & 35 & 85.4 & 24 & 82.8 & \\
\hline Type of pain (Always) & 21 & 51.2 & 05 & 17.2 & 0.004 \\
\hline Type of pain (Random) & 20 & 48.8 & 24 & 82.8 & \\
\hline Pain at night & 09 & 22.0 & 06 & 20.7 & 0.899 \\
\hline Pain affecting playing & 24 & 58.5 & 26 & 89.7 & 0.005 \\
\hline Pain affecting studying & 19 & 46.3 & 19 & 65.5 & 0.113 \\
\hline
\end{tabular}

${ }^{*}$ Chi-square test

Table 2 : Comparison of dental caries between the intervention and control groups

\begin{tabular}{|l|c|c|}
\hline & $\begin{array}{l}\text { Control } \\
\text { group } \\
(\mathrm{N}=161)\end{array}$ & $\begin{array}{l}\text { Intervention group } \\
(\mathrm{N}=162)\end{array}$ \\
\hline Number of students with dental caries (percentage) & $78(48.4)$ & $131(80.9)$ \\
\hline $\begin{array}{l}\text { Mean number of teeth with dental caries (Standard } \\
\text { Deviation) }\end{array}$ & $0.74(0.90)$ & $1.35(0.88)$ \\
\hline
\end{tabular}


Table 3: Association between sodium fluoride varnish application and new dental caries at different time points

\begin{tabular}{|l|r|r|l|}
\hline Period & $\begin{array}{l}\text { Mean number of new dental caries in the } \\
\text { Intervention Group }\end{array}$ & $\begin{array}{l}\text { Mean number of new dental caries in } \\
\text { the Control Group }\end{array}$ & $\begin{array}{l}\mathbf{p} \\
\text { value }^{\#}\end{array}$ \\
\hline $\begin{array}{l}2015 \\
\text { May }\end{array}$ & 0.94 & 1.79 & $<0.001$ \\
\hline 2015 & 1.69 & 1.77 & $<0.001$ \\
Dec & 1.50 & 1.97 & $<0.001$ \\
\hline $\begin{array}{l}2016 \\
\text { Nov }\end{array}$ & & & 1.001 \\
\hline
\end{tabular}

\#Mann-Whitney U test

Table 4: Independent association between vanish intervention and new dental caries

\begin{tabular}{|c|r|l|l|}
\hline Period & $\boldsymbol{\beta}$ coefficient & $95 \% \mathrm{Cl}$ & p value \\
\hline May 2015\# & & & \\
\hline Intervention & -0.84 & $-1.24--0.45$ & $<0.001$ \\
\hline Control & 1 & & \\
\hline Dec 2015\# & & & \\
\hline Intervention & 0.09 & $-0.45-0.64$ & 0.733 \\
\hline Control & 1 & & \\
\hline Nov 2016\# & & & \\
\hline Intervention & -0.49 & $-1.0--0.28$ & 0.043 \\
\hline Control & 1 & & \\
\hline
\end{tabular}

\# Other independent variables controlled in the model: Age, mothers education status, household income status, presence of toothach at the baseline

\section{Supplementary Files}

This is a list of supplementary files associated with this preprint. Click to download.

- CONSORT2010ChecklistFV.pdf 